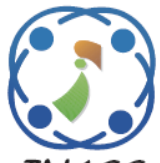

\title{
Modified Headed Social Force Model Based on Hybrid Velocity Obstacles for Mobile Robot to Avoid Disturbed Groups of Pedestrians
}

\author{
Muhammad Fuad ${ }^{1,2 *}$ \\ Trihastuti Agustinah ${ }^{1 *}$ \\ Djoko Purwanto ${ }^{1}$ \\ ${ }^{I}$ Department of Electrical Engineering, Institut Teknologi Sepuluh Nopember, Surabaya, East Java, Indonesia \\ ${ }^{2}$ Faculty of Engineering, University of Trunojoyo Madura, Bangkalan, East Java, Indonesia \\ *Corresponding author's Email: trihastuti@ee.its.ac.id
}

\begin{abstract}
The need for mobile robots that can operate in the human environment is increasing during the Covid-19 pandemic. To accomplish this task, robot navigation must be supported by collision avoidance to maintain human safety and comfort. Collision avoidance methods generally only maintain a safe distance from surrounding objects. Some methods that provide comfort still have difficulty in overcoming pedestrians that move with unexpected direction, changing speed, and unknown trajectories. This paper proposes Hybrid Velocity Obstacle-based modified Headed Social Force Model (HVO-based modified HSFM) to avoid disturbed groups of pedestrians while navigating in the complex and dense workspace. HVO is used to calculate linear and angular velocities to avoid obstacles with nonlinear trajectories. The linear velocity that computed by $\mathrm{HVO}$ acts as desired velocity for generating target force of modified HSFM that drive robot to target location. While interaction force of modified HSFM that guide robot to circumvent obstacles is determined by static and moving objects in the surrounding of robot. The angular velocity from HVO is used to produce steering command to avoid collision. For evaluating the proposed method, several simulation scenarios had been run by implementing HVO-based modified HSFM into a two-wheeled differential-steering mobile robot that navigate in the indoor human environment. The results show that our approach is capable to avoid collision by maintaining safety and comfort with disturbed groups of pedestrians with average value 0.14 of Threat Level Index (TLI) from two scenarios. It is envisioned that proposed method can be implemented into real transport robot that operate in human environment.
\end{abstract}

Keywords: Differential-steering wheeled mobile robot, Disturbed groups of pedestrians, Headed social force model, Hybrid velocity obstacles, Collision avoidance, Nonlinear trajectories.

\section{Introduction}

The world regular living has changed because of Corona virus (COVID-19) pandemic. It is influencing how to give our services and how to get our needs. This pandemic is causing us to reconsider the safety protocols of everyday processes by prioritizing public health. Safer work environments can be constructed by utilizing robotics. Some areas that can use robots to fight this global disease are cleaning, manufacturing, logistics, healthcare, and telepresence [1]. For responding to potential supplychain disruptions, robot can play an essential part of daily activities by taking parts as reliable logistics fleet. Mobile robots are used to deliver packages to their target destinations [2]. To be able to do this, the robot must be equipped with the ability to navigate near humans by maintaining safety and comfort [3]. Navigation can maintain these factors because of the support of collision avoidance.

There are several collision avoidance approaches proposed in the literatures. Artificial Potential Field (APF) based on attractive and repulsive force that has been modified were implemented for collision avoidance strategy of AUV and mobile robot respectively in [4] and [5]. Some improvements on Vector Field Histogram (VFH) were applied to direct the obstacle avoidance movement of mobile robot with laser scanner [6] and microrobot [7]. An effort to integrate LIDAR sensor to provide steering command on Velocity Obstacles (VO) was explored 
in [8] to support navigation function for Autonomous Indoor Vehicle (AIV).

An application of $\mathrm{VO}$ into path planning of autonomous vehicles guidance for avoiding static lane edges, crossing pedestrian and other vehicles was investigated in [9]. Reciprocal Velocity Obstacles (RVO) was used to support Deep Reinforcement Learning for avoiding agents collision in a crowd navigation $[10,11]$. For handling collision avoidance of mobile robot that navigate in close proximity to human and other multi modal moving objects, Hybrid Velocity Obstacles (HVO) was presented in [12]. Although these algorithms can maintain safety, they still need to preserve human comfort so that the robot can operate in the human environment appropriately.

Another approach considers human psychological comfort for single human and group of pedestrians. Intentionally, people tend to walk together as a group. Therefore, it is necessary to explore how to pay attention to group comfort. In order to enhance the realism of generated trajectories, Headed Social Force Model (HSFM) was introduced in [13] by considering the behavior of people while walking in a group.

Generally, these previous studies had assumption that obstacles move with constant velocities. Research to explore group of pedestrians as uncooperative moving obstacles that changing their direction or speed is still rare and it need to be developed. In reality, group of walking humans can have acceleration or deceleration because of they had been disturbed by some condition. Hence, no previous approaches had considered nonlinearity in the trajectory of dynamic changing moving group of humans.

This paper aims to develop collision avoidance system for avoiding disturbed groups of pedestrians with respect to kinematics and dynamics of mobile robot by presenting HVO-based modified HSFM.

The rest of this paper is organized as follows. Contributions of this paper are described in section 2 . The state of the arts in collision avoidance are reviewed in section 3. Section 4 presents disturbed groups of pedestrians. Section 5 defines the proposed collision avoidance system used in this research. Section 6 evaluates the proposed approach in simulations with two scenarios. Section 7 concludes this paper.

\section{Contributions}

The main contributions of this paper are

- Local path planner combines Pure Pursuit and HVO-based modified HSFM to handle path following and collision avoidance. This research contributes to utilizing $\mathrm{HVO}$ for providing steering command and desired velocity of modified HSFM to avoid static and moving humans that exist in the vicinity of robot by maintaining human safety and comfort with respect to kinematics and dynamics of mobile robot. Unlike previous studies, this research considers maintaining safety and comfort of more complete obstacles modalities including static pedestrians, individual pedestrian, normal groups of pedestrians, and disturbed groups of pedestrians. This proposed collision avoidance system is evaluated using real and rare problems.

- Global path planner of proposed collision avoidance system exploits Probabilistic Road Map (PRM) planner to generate random desired path from initial to target pose for each moving object in simulation. This method application is unique and more realistic compare to previous research which based on a fixed set of waypoints.

- Structure of mobile robot navigation system adopts switching strategy between path following and collision avoidance to generate linear and angular velocity reference. By separating global path planner and local path planner, navigation structure becomes more detailed and computation is faster because the system does not have to do replanning when environment change. It also differs from other investigations which based solely on velocity or orientation for controlling mobile robot movement. The output of this strategy, linear and angular velocities references, can be simply applied to real robot.

\section{Related works}

Comparison of several collision avoidance approaches based on modalities, kinematics / dynamics, control, and their impact on safety / comfort are summarized in Table 1.

An interesting strategy to separate global path planner from local path planner was explored in [8]. Probabilistic Road Map (PRM) planner was used as global path planner. While Pure Pursuit (PP) and Modified Steering Velocity Obstacles (MSVO) were employed as local path planner by relying on sensor reading for planner selection. A related indoor navigation system was presented in [12] by replacing MSVO with HVO that acts as local path planner for handling multi modal moving obstacles. This paper adopts the simplicity of navigation structure as proposed in [8] and [12] by substituting MSVO and HVO with HVO-based modified HSFM as collision 
Table 1. Comparison of some studies on collision avoidance

\begin{tabular}{|c|c|c|c|c|}
\hline Approach & Obstacles Modalities & $\begin{array}{c}\text { Kinematics / } \\
\text { Dynamics }\end{array}$ & Control & Safety / Comfort \\
\hline APF [4] & Static and moving & No & $v$ & Safety \\
\hline APF [5] & Static & No & $\theta$ & Safety \\
\hline VFH[6] & Static and moving & No & $\theta$ & Safety \\
\hline VFH [7] & Static and moving & Kinematics & $\theta$ & Safety \\
\hline $\begin{array}{l}\text { Modified-steering VO } \\
{[8]}\end{array}$ & Static & Kinematics & $v, \omega$ & Safety \\
\hline VO [9] & $\begin{array}{l}\text { Static and moving, single } \\
\text { pedestrian }\end{array}$ & Kinematics & $v$ & Safety \\
\hline RVO [10],[11] & $\begin{array}{l}\text { Static, dynamic, group of } \\
\text { agents }\end{array}$ & No & $v$ & Safety \\
\hline HVO [12] & $\begin{array}{l}\text { Static, nonlinear } \\
\text { trajectory moving } \\
\text { objects, single pedestrian, } \\
\text { group pedestrian }\end{array}$ & Kinematics & $v, \omega$ & Safety \\
\hline HSFM [13] & Group pedestrian & $\begin{array}{l}\text { Kinematics/ } \\
\text { dynamics of } \\
\text { pedestrian }\end{array}$ & $v$ & Comfort \\
\hline $\begin{array}{l}\text { HVO-based Modified } \\
\text { HSFM, the proposed } \\
\text { method }\end{array}$ & $\begin{array}{l}\text { Disturbed groups of } \\
\text { pedestrians }\end{array}$ & $\begin{array}{l}\text { Kinematics/ } \\
\text { dynamics }\end{array}$ & $v, \omega$ & Safety, Comfort \\
\hline
\end{tabular}

avoidance method in local path planner. This strategy generates the desired path once it gets information about initial and target pose.

This global path is calculated by considering environment boundary, static, and moving objects in the surrounding of robot. Computation burden can be reduced by doing global path planning only once. The other advantage of this proposed switching strategy is that the system produces linear and angular velocities with respect to kinematics and dynamics of mobile robot which can be directly applied to the real plant.

A fixed set of waypoints was utilized in [9] to direct pedestrian toward destination position. While in [5] robot was commanded to cross from one initial point to target point. An interesting way to generate random set of desired paths by applying PRM was explored in [8] and [12]. This probabilistic algorithm was implemented in all moving objects that exist in the environment to be closer to reality. This approach is taken in this study in order to make the simulation more realistic because humans move with their random trajectory [14].

Several studies in collision avoidance considered to maintain safe distance with static objects only such as in [5] and [8]. The others manage to avoid static and moving objects safely $[4,6,7,9]$. Some of these studies more concern to keep comfort of pedestrian as individual [9] and [12]. As in [15], it stated that humans tend to move in group. To answer this requirement, other studies added comfort of group as their duty to maintain, such as examined in [10-13].

In general, these studies assume that the speed and direction of pedestrians, both individuals and groups, are always constant except in [12]. The speed and direction change in [12] were happened to represent human avoiding behaviour. This behaviour can also appear in catch-up situation. This situation occurred among groups of pedestrians with the same directions at the narrow corridor when the front group is overtaken by the rear group. According to [16], people walk faster not only because of motivation to overtake but rather it occurs when personal space is invaded by disturbance. Research in pedestriancollision avoidance for mobile robot that involve groups of pedestrians with changeable velocities triggered by distraction is still rare. This research proposes a method to avoid group of moving 
objects with fluctuating speed and direction. In this paper, people that walk with changes in speed and direction because of some disturbances are called disturbed groups of pedestrians.

\section{Disturbed groups of pedestrians}

Mobile robot that operates in indoor environment must have the capability to avoid collision with static objects, static human, and pedestrians. This scenario is illustrated in Fig. 1.

This mobile robot has to transport some stuff to certain target location by providing safety and comfort of human in the vicinity of robot. The dashed boundary of each grey objects show the area of a safety and comfort standard that must be avoided by robot. An indoor human environment, such as hall, may contain static and moving humans. Static humans comprise of human-object interaction and group of static humans in face-to-face formation. Moving humans involve single pedestrian, group of pedestrians with normal velocity, and disturbed group of pedestrians.

Disturbed groups of pedestrians that have been a concern of this study are type of pedestrians that walk with faster velocities than normal pedestrians. Their characters are the same with single pedestrian in that they move with changeable velocities and nonlinear trajectories. Human properties as individual is illustrated in Fig. 2.

Individual human can be described by human pose in $\mathrm{X}-\mathrm{Y}$ coordinate $\left[x_{h i}, y_{h i}, \psi_{h i}\right]^{T}$, velocity $v_{h i}$, acceleration $a_{h i}$ and radius of the- $i^{\text {th }}$ individual human body $r_{h i}$ that can be affected by arms reached or helping devices as formulated as follows:

$$
\mathbf{S}_{h i}=\left[x_{h i}, y_{h i}, \psi_{h i}, v_{h i}, a_{h i}, r_{h i}\right]^{T}
$$

This representation of individual human is different from previous study in [12] that acknowledged obstacle object in the form of human as point-mass that can move in arbitrary direction $\left[\begin{array}{lll}x_{o} & y_{o} & \psi_{o}\end{array}\right]^{T}$. Furthermore, this study exploits more flexible property to symbolize the outer border of human body by using the radius of body coverage $r_{h i}$. In real life, the radius of human body coverage can be varying. It should not only be measured by position of their hands, but also may include some devices that people with disabilities used such as cane, crutches or wheelchair. Therefore, the outer border of human body is measured from the center of human body to the position of hands or other helping devices that denoted by $r_{h i}$ that can be affected by arms reached or helping devices as formulated as follows:

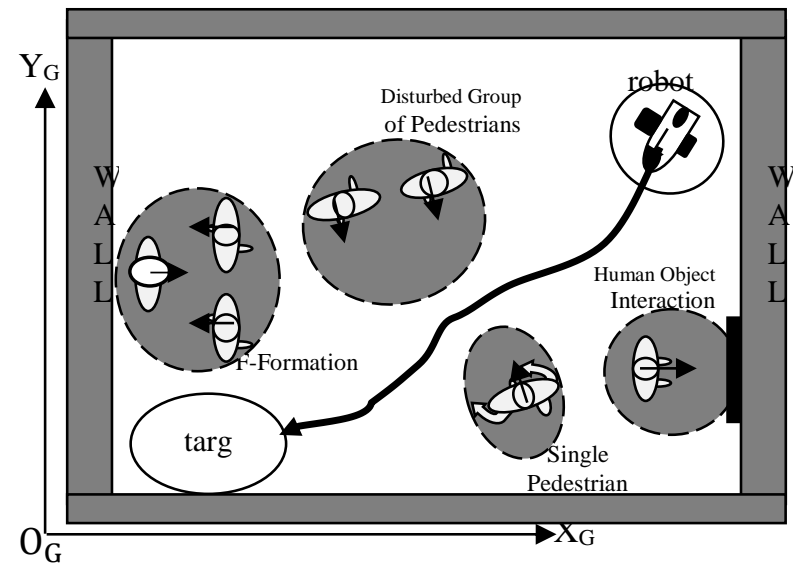

Figure.1 Interaction between mobile robot and disturbed group of pedestrians in indoor human environment

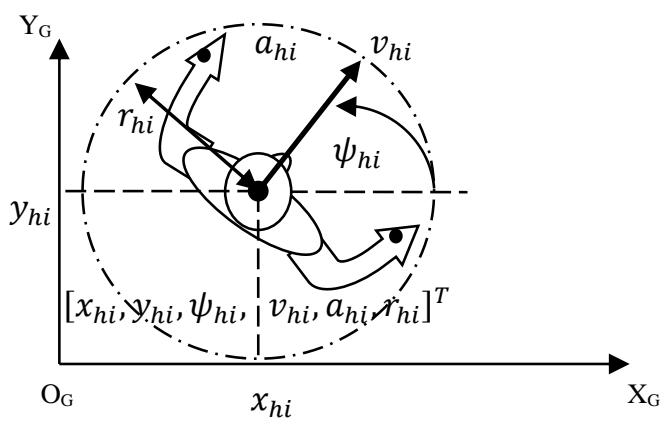

Figure.2 State of individual human

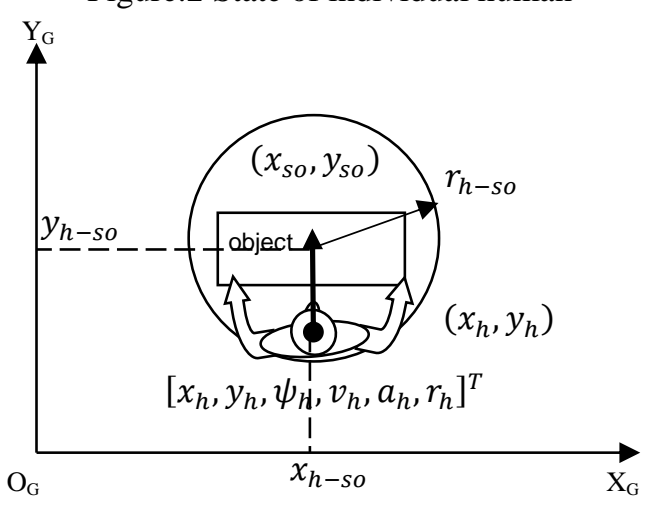

Figure.3 Interaction between human and static object

Human can interact with static object in the environment. Some static objects in the environment are information board, paint, and screen display. Fig.3 illustrates human-object interaction (HOI).

This interaction forms a state as follows:

$$
\mathbf{S}_{h-s o_{m}}=\left[x_{h-s o_{m}}, y_{h-s o_{m}}, r_{h-s o_{m}}\right]^{T}
$$

where $\left(x_{h-s o_{m}}, y_{h-s o_{m}}\right)$ represents the $m^{\text {th }}$ center point of HOI area. While radius of this area is denoted by $r_{h-s o_{m}}$. Centre point and radius of HOI area can 
be calculated using following equations:

$$
\begin{aligned}
& \left(x_{h-s o_{m}}, y_{h-s o_{m}}\right)=\left(x_{s o}, y_{s o}\right) \\
& r_{h-s o_{m}}=\sqrt{\left(x_{s o}-x_{h}\right)^{2}+\left(y_{s o}-y_{h}\right)^{2}}
\end{aligned}
$$

It assumes that location of static object $\left(x_{s o}, y_{s o}\right)$ is positioned at the center of HOI area. Therefore, center point of this interaction should be at the position of the static object itself.

Each individual human is assumed to has four distance zones such as depicted in Fig. 4. This study proposes ellipse-based regions for realizing these zones. These regions have concern on forward and backward direction more than on side direction. It is useful especially when human move in narrow space. In relation to others, human tends to involve in social interaction group. Social interaction between human with immobile condition forms a face-to-face formation. This formation is known as F-Formation [19]. In connection with human safety and comfort, there are three important aspects that have to be concerned in F-Formation such displayed in Fig. 5. $\mathrm{O}$-space is the depest area in this formation. This area represents a personal zone of each members of FFormation. While p-space denotes an area which is occupied by human for interaction. Whereas the area outside $\mathrm{p}$-space that has to be concerned as the source of safety and comfort, is denoted as r-space. Social zone of each member of formation is represented by r-space.

The geometry of human locomotion on this study is approximated by non-holonomic model such as explored in [17] because most of time humans tend to move forward.

Human locomotion based on non-holonomic model can be described as follows:

$$
\left[\begin{array}{c}
\dot{x}_{h} \\
\dot{y}_{h} \\
\dot{\psi}_{h}
\end{array}\right]=\left[\begin{array}{cc}
\cos \psi_{h} & 0 \\
\sin \psi_{h} & 0 \\
0 & 1
\end{array}\right]\left[\begin{array}{c}
v_{h} \\
\omega_{h}
\end{array}\right]
$$

where human velocity $\mathbf{v}_{h}=\left[\begin{array}{ll}v_{h} & \omega_{h}\end{array}\right]^{T}$. Pose of shoulder midpoint is denoted by $\left[x_{h}, y_{h}, \psi_{h}\right]^{T}$ as representation of human in global coordinate. This equation can construct configuration transition of human from body to global coordinate.

Forward kinematics model of non-holonomic human locomotion is represented as follows:

$$
\left[\begin{array}{c}
v_{h} \\
\omega_{h}
\end{array}\right]=r_{h}\left[\begin{array}{cc}
1 / 2 & 1 / 2 \\
1 / d_{h} & -1 / d_{h}
\end{array}\right]\left[\begin{array}{c}
\dot{\theta}^{R}{ }_{h} \\
\dot{\theta}^{L}{ }_{h}
\end{array}\right]
$$

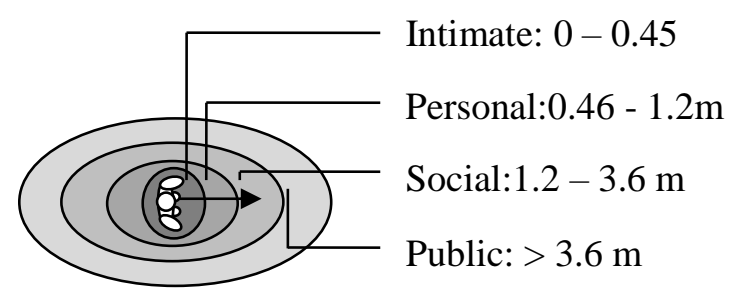

Figure.4 Interpersonal distance of individual human

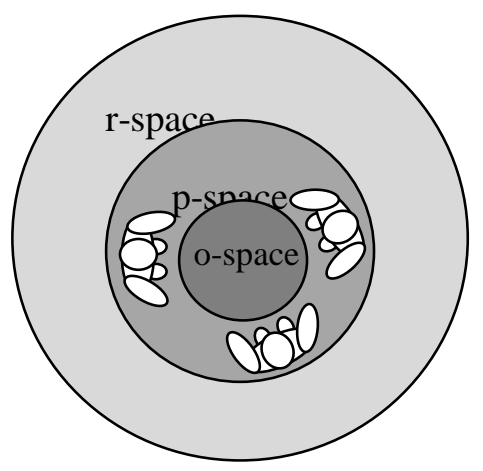

Figure.5 Important spaces in FFormation

where human footstep $r_{h}$, body diameter $d_{h}$, speed of human footstep $\dot{\mathbf{q}}_{h}=\left[\begin{array}{ll}\dot{\theta}^{R}{ }_{h} & \dot{\theta}^{L}{ }_{h}\end{array}\right]^{T}$ are used to steer human movement by affecting its linear and angular velocity $\mathbf{v}_{h}$.

In other way, inverse kinematics can be used to obtain speed of human footstep $\dot{\mathbf{q}}_{h}$ from its linear and angular velocity $\mathbf{v}_{h}$ as defined as follows:

$$
\left[\begin{array}{c}
\dot{\theta}^{R}{ }_{h} \\
\dot{\theta}_{h}^{L}
\end{array}\right]=\frac{1}{r_{h}}\left[\begin{array}{cc}
1 & d_{h / 2} \\
1 & -d_{h / 2}
\end{array}\right]\left[\begin{array}{l}
v_{h} \\
\omega_{h}
\end{array}\right]
$$

Human as individual or in a group tends to walk with certain velocity. This velocity can be changed because of influence of their surrounding objects. This study uses HSFM [13] to model forces that have influence on human movement. Based on HSFM, change on human velocity can be calculated as follows

$$
\begin{gathered}
a_{h}(k)=\frac{1}{m_{h}} \mathbf{F}_{h}^{H S F M}(k) \\
v_{h}^{*}(k)=v_{h}(k)+a_{h}(k) T
\end{gathered}
$$

where acceleration of human $a_{h}(k)$ can be obtained by providing human body mass $m_{h}$ and total force $\mathbf{F}_{h}^{H S F M}(k)$ that act on human. The actual velocity of pedestrian is denoted by $v_{h}(k)$. Discrete 
time interval while acceleration $a_{h}(k)$ happen is symbolized by $T$. The new actual velocity of pedestrian is expressed by $v_{h}^{*}(k)$.

This approach consists of attractive force $\mathbf{F}_{h-t a r}^{\text {att }}$ and some repulsive forces $\mathbf{F}_{h-o b s}^{r e p}$ as formulated as follows:

$$
\mathbf{F}_{h}^{H S F M}=\mathbf{F}_{h-t a r}^{a t t}+\sum \mathbf{F}_{h-o b s}^{r e p}
$$

The location of interest results attractive force $\mathbf{F}_{h-\text { tar }}^{\text {att }}$ that motivate human to approach. Timeconstant $K_{h}^{v}$ is needed for moving from current actual velocity $v_{h}^{a c t}$ to desired velocity $v_{h}^{\text {des }}$. Equation to obtain attractive force is described as follows:

$$
\mathbf{F}_{h-t a r}^{\text {att }}=K_{h}^{v}\left(v_{h}^{\text {des }}-v_{h}^{a c t}\right)
$$

The sum of repulsive force $\sum \mathbf{F}_{h-o b s}^{\text {rep }}$ are affected by static obstacle objects $\mathbf{F}_{h-s o}^{r e p}$ and other static or moving human $\mathbf{F}_{h-o h}^{r e p}$ as described as follows:

$$
\sum \mathbf{F}_{h-o b s}^{r e p}=\mathbf{F}_{h-s o}^{r e p}+\mathbf{F}_{h-o h}^{r e p}
$$

In general, repulsive force is defined as follows:

$$
\begin{aligned}
& \mathbf{F}_{h-o b s}^{r e p} \\
& =K_{h}^{s t r} \exp \left(\frac{\left(r_{h-o b s}-d_{h-o b s}\right)}{K_{h}^{r n g}}\right) \boldsymbol{n}_{h-o b s} \\
& +K_{h}^{c m p} \mathrm{~g}\left(r_{h-o b s}-d_{h-o b s}\right) \boldsymbol{n}_{h-o b s} \\
& +K_{h}^{f r i} \mathrm{~g}\left(r_{h-o b s}\right. \\
& \left.-d_{h-o b s}\right) \Delta v_{o b s-h}^{t} \mathbf{t}_{h-o b s}
\end{aligned}
$$

Repulsive force $\mathbf{F}_{h-o b s}^{r e p}$ that push human to evade, consists of $K_{h}^{s t r}$ as a constant that represents strength. $K_{h}^{r n g}$ states a constant that defines range of social interaction. If human and objects in its environmentthat assumed to be circle then total radius of boths are declared as $r_{h-o b s}$ and distance between boths center point are notated as $d_{h-o b s}$. Normalization vector that pointing from obstacle objects to human is denoted by $\boldsymbol{n}_{h-o b s}$. Constant of body compression and friction are described by $K_{h}^{c m p}$ and $K_{h}^{f r i}$ respectively. Tangential velocity difference is represented by $\Delta v_{o b s-h}^{t}$. Tangential direction is expressed by $\mathbf{t}_{h-o b s}$. Repulsive force for each term will be described in the next section.

Beside gathering in static mode as known as FFormation, human can also form social groupings comprise of two or more individuals to move together.
Group of humans that walking together is known as mobile formation [20]. Groups of pedestrians may manage to start or stop walking together at different times, maintain their relative movement at different speeds, and change mobile trajectories.

Human as individual pedestrian or human in groups of pedestrians usually walk in normal speed $1.3 \mathrm{~m} / \mathrm{s}$ with their random path. This study applies PRM to generate random set of desired paths of each moving object in the environment including object that represent walking human or pedestrian.

There are several inputs need to be provided to generate random path with PRM as illustrated in Fig. 6. Environmental map of robot workspace is the first input that act as the basis information to generate some nodes randomly. A roadmap of interconnected nodes can be created based on number of nodes and distance between nodes. By supplying initial and target position of each moving object as the second and the third inputs, a nonlinear path $\left[x_{h}(k), y_{h}(k)\right]$ of interrelated nodes from start to goal point of this roadmap can be produced. Preprocessing phase of PRM that used in this study is described in Algorithm 1. Different with previous studies in [8] and [12], this study proposes to modify distance $r$ that connect each node to be a function of the number of node samples $n$ by introducing $\operatorname{Scaled}(n)$. This function replaces the way of previous studies that define distance $r$ as constant value with $\log (n)$ that proportionally relate to number of node samples.

The result of two separate running sessions of PRM application in generating random path with the same pairs of initial and target position are depicted in Fig. 7.

The upper one is the result of original PRM. The bottom is the result of modified PRM. These results are produced from the same amount of node samples. Speed and direction of pedestrians can change when obstacles are disturbed physically. Therefore, repulsive forces $\sum \mathbf{F}_{h-o b s}^{r e p}$ affect this condition. Their speed and trajectories can vary also because of psychological disturbance. Thus, attractive force $\mathbf{F}_{h-\text { tar }}^{\text {att }}$ has influence in this situation.

This study introduces a new problem facing by mobile robot that operate in human environment in the form of disturbed groups of pedestrians that change their speed and direction because of or psychologically disturbance. Our modified HSFM is not designed to proactively anticipate speed and direction change of pedestrians. This research proposes to integrate HVO into modified HSFM to avoid disturbed groups of pedestrians by maintaining safety and comfort of static or moving human in the 


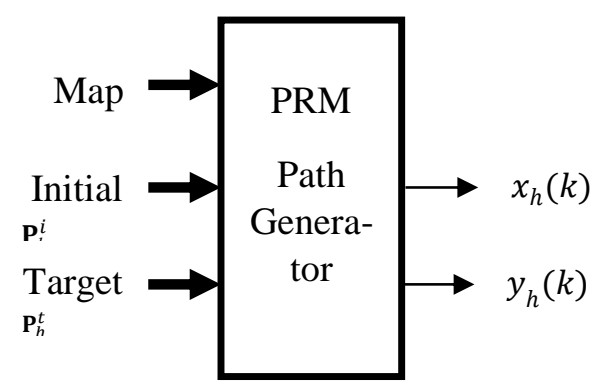

Figure.6 PRM is used to generate random path of walking human

surrounding of mobile robot. Details of collision avoidance system with HVO-based modified HSFM will be described in the following section.

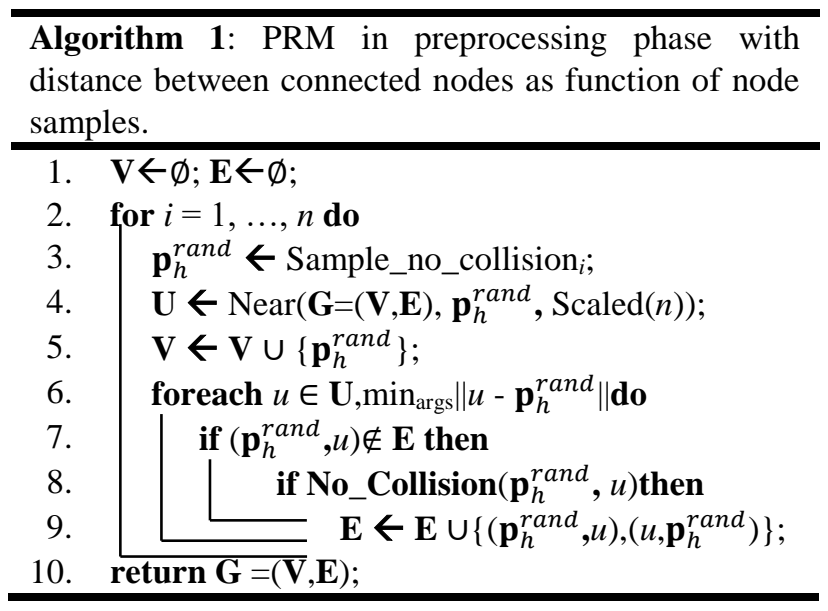

\section{Proposed collision avoidance system}

Collision avoidance system is designed by considering kinematics and dynamics of mobile robot with respect to safety and comfort distance of static human, single pedestrian, group of pedestrian, and disturbed groups of pedestrians in the robot environment.

\subsection{Structure of collision avoidance system}

This study proposes indoor navigation system based on trajectory tracking equipped with collision avoidance system with respect to kinematics and dynamics of two wheeled differential-steering mobile robot such as described in Fig. 8.

Desired mobile robot pose that acts as reference, $\mathbf{P}_{d}=\left[\begin{array}{lll}x_{d} & y_{d} & \psi_{d}\end{array}\right]^{T}$, is generated by PRM path planner. It connects initial to target position by a set of waypoints that randomly generated. The waypoints have to be tracked by mobile robot. Actual mobile robot pose is denoted by $\mathbf{P}_{r}=$ $\left[\begin{array}{lll}x_{r} & y_{r} & \psi_{r}\end{array}\right]^{T}$. Obstacle pose is expressed by $\mathbf{P}_{o}=$ $\left[\begin{array}{lll}x_{o} & y_{o} & \psi_{o}\end{array}\right]^{T}$. Desired robot pose, human pose,
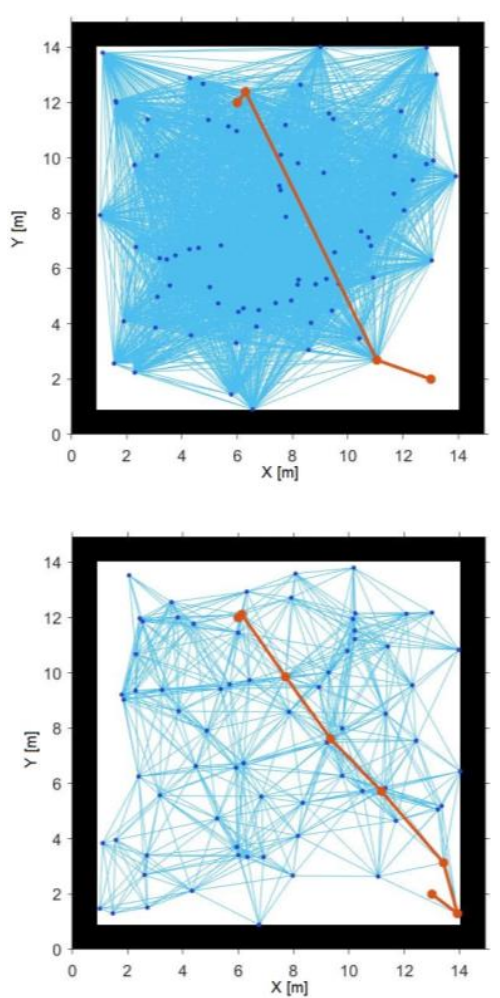

Figure.7 Random path of original and modified PRM

and actual robot pose are used to calculate velocity command either for tracking or collision avoidance purposes.

Humans that exist in the environment includes static human, moving human as single pedestrian, group of pedestrians, and disturbed groups of pedestrians as described in the previous section are acted as obstacles. Although humans are included in obstacles, robot have to maintain safety and comfort of humans while it navigates from initial to target position. Standard of safety and comfort distance are presented in the next sub sections.

\subsection{Standard of safety and comfort distance}

Obstacles radii are measured from their center to the outer border of each objects. Beyond this dimension, there are several layers of interpersonal distances of human as described in previous section that need attention in order to maintain safety and comfort. Violation in certain distance will threaten their physical safety. Disturbance in specified distance will distract their psychological comfort.

This research proposes Threat Level Index (TLI) to evaluate the safety and comfort distance for each

object existing in the environment as follows:

$$
T L I=e^{-\frac{d_{r-o}}{\sigma}}
$$






Figure.8 Structure of indoor navigation system

This evaluation index comparing between the standard deviation $\sigma$ and actual distance of each object to mobile robot $d_{r-o}$. The standard deviation $\sigma$ is defined at $0.65 \mathrm{~m}$. This parameter value provides enough space for mobile robot to manoeuvre. Actual distance between mobile robot and objects $d_{r-o}$ is determined by using Euclidean distance. A safety standard is defined at $0.5 \mathrm{~m}$ or equal with 0.46 of TLI. A comfort standard is stated at $0.8 \mathrm{~m}$ or equal with 0.29 of TLI. These values represent close and far phase of personal distance [18] respectively. They are also suitable with physical distancing program in pandemic era. The smaller $T L I$ value means the safer and more comfort condition are. Object safety and comfort is threatened when $T L I$ value greater than 0.46 .

\subsection{Kinematics and dynamics of mobile robot}

This research uses a two wheeled differentialsteering mobile robot to deliver a task from its initial to target position in indoor environment. The robot posture in the Cartesian coordinate system is depicted in Fig. 9. The width of robot is expressed as $d$. The centre of left-right wheel axis is denoted as $C$.

The centre of mass is described as $G$. This point represents robot in the global X-Y coordinate. Left and right motors rotate wheels with diameter $2 r$. Mobile robot pose and its configuration transition are denoted as follows:

$$
\begin{aligned}
& \mathbf{P}_{r}=\left[\begin{array}{lll}
x_{r}, & y_{r}, & \psi_{r}
\end{array}\right]^{T}, \\
& \dot{\mathbf{P}}_{r}=\left[\begin{array}{lll}
\dot{x}_{r}, & \dot{y}_{r}, & \dot{\psi}_{r}
\end{array}\right]^{T}
\end{aligned}
$$

where robot position is expressed by $\left[x_{r}, y_{r}\right]^{T}$ and orientation is symbolized by $\psi_{r}$. Configuration transition of robot pose from body to global coordinate is stated as follows:

$$
\left[\begin{array}{c}
\dot{x}_{r} \\
\dot{y}_{r} \\
\dot{\psi}_{r}
\end{array}\right]=\left[\begin{array}{cc}
\cos \psi_{r} & 0 \\
\sin \psi_{r} & 0 \\
0 & 1
\end{array}\right]\left[\begin{array}{l}
v_{r} \\
\omega_{r}
\end{array}\right]
$$

where mobile robot velocity $\mathbf{v}_{r}=\left[\begin{array}{ll}v_{r} & \omega_{r}\end{array}\right]^{T}$ causes the change in its pose in global coordinate $\dot{\mathbf{P}}_{r}=\left[\dot{x}_{r}, \dot{y}_{r}, \dot{\psi}_{r}\right]^{T}$. Forward kinematics model is defined as follows:

$$
\left[\begin{array}{l}
v_{r} \\
\omega_{r}
\end{array}\right]=r\left[\begin{array}{cc}
1 / 2 & 1 / 2 \\
1 / d & -1 / d
\end{array}\right]\left[\begin{array}{c}
\dot{\theta}^{R}{ }_{r} \\
\dot{\theta}^{L}{ }_{r}
\end{array}\right]
$$

where wheel speeds $\quad \dot{\mathbf{q}}_{r}=\left[\begin{array}{ll}\dot{\theta}^{R}{ }_{r} & \dot{\theta}^{L}{ }_{r}\end{array}\right]^{T}$ are employed to drive mobile robot based on its wheel radius $r$ and width of robot $d$ by affecting its linear $v_{r}$ and angular velocity $\omega_{r}$.

Wheel speeds $\dot{\mathbf{q}}_{r}$ can be obtained from linear and angular velocity $\mathbf{v}_{r}$ by applying inverse kinematics as follows:

$$
\left[\begin{array}{c}
\dot{\theta}^{R}{ }_{r} \\
\dot{\theta}^{L}{ }_{r}
\end{array}\right]=\frac{1}{r}\left[\begin{array}{cc}
1 & d / 2 \\
1 & -d / 2
\end{array}\right]\left[\begin{array}{c}
v_{r} \\
\omega_{r}
\end{array}\right]
$$

Eulerian integration is used to update robot pose as follows: 


$$
\left[\begin{array}{l}
x_{r}(k+1) \\
y_{r}(k+1) \\
\psi_{r}(k+1)
\end{array}\right]=\left[\begin{array}{l}
x_{r}(k) \\
y_{r}(k) \\
\psi_{r}(k)
\end{array}\right]+k T\left[\begin{array}{c}
\dot{x}_{r}(k) \\
\dot{y}_{r}(k) \\
\dot{\psi}_{r}(k)
\end{array}\right]
$$

Dynamic model of mobile robot can be expressed using Newton-Euler as follows:

$$
\begin{gathered}
\mathbf{F}_{r}=m_{r} a_{r}=m_{r} \dot{v}_{r} \\
\mathbf{N}_{r}=I_{r} \dot{\omega}_{r} \\
\dot{v}_{r}=\frac{1}{m_{r} r}\left(\tau_{R}+\tau_{L}\right) \\
\dot{\omega}_{r}=\frac{d}{I_{r} r}\left(\tau_{R}-\tau_{L}\right)
\end{gathered}
$$

where total force that acts on robot at its center of gravity is represented by $\mathbf{F}_{r}$, total torque is denoted by $\mathbf{N}_{r}$, mass of robot body is symbolized by $m_{r}$, acceleration is declared as $a_{r}$, inertia of robot is stated as $I_{r}$, torque of right and left motor are described as $\tau_{R}$ and $\tau_{L}$ respectively.

\subsection{LIDAR Sensor}

Mobile robot was equipped with simulated LIDAR (light detection and ranging) scan. It has max sensing range of $20 \mathrm{~m}$. The horizontal field of view is $360^{\circ}$ with resolution $12^{\circ}$ as depicted in Fig. 10. It generates ranges reading $\mathbf{d}_{s}$ that measured from the sensor to obstacles in the environment with respect to its specification.

\subsection{Velocity command selection}

Velocity command selects to activate one of two method between tracking and collision avoidance for generating appropriate velocity by using Algorithm2 . It is switched based on lidar scan $\mathbf{d}_{s}$ at objects in the surrounding of robot. Collision avoidance will activate if LIDAR scan ranges reading $\mathbf{d}_{s}$ less than alert distance $\mathbf{d}_{a}$.

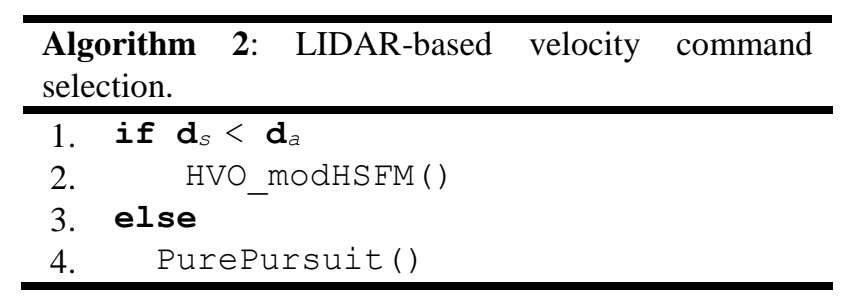

\subsection{Pure pursuit velocity command}

For path following purposes, a tracking method named Pure Pursuit (PP) is used to guide robot movement from initial to target position. These two values are a part of waypoints $\mathbf{P}_{d}$ as one of parameters required by PP. The other parameters are look ahead distance, desired linear velocity, and maximum angular velocity. Based on these parameters, PP computes the linear $v_{c m d}^{p f}$ and angular velocity $\omega_{c m d}^{p f}$ as velocity command $\mathbf{v}_{c m d}^{p f}$ for path following commands to reach a look-ahead point in front of the robot by giving the actual pose of the robot $\mathbf{P}_{r}$ as input.

\subsection{HVO-based modified HSFM velocity command}

This research proposes a modified version of HSFM model based on HVO for collision avoidance purposes with respect to safety and comfort of human in the surrounding of robot. HSFM that is used by mobile robot $\mathbf{F}_{r}^{H V O}$-modHSFM consists of some repulsive forces $\mathbf{F}_{r-o b s}^{\text {rep }}$ and attractive force $\mathbf{F}_{r-t a r}^{\text {att }}$ as formulated as follows:

$$
\mathbf{F}_{r}^{H V O-\operatorname{modHSFM}}=\sum \mathbf{F}_{r-o b s}^{r e p}+\mathbf{F}_{r-t a r}^{a t t}
$$

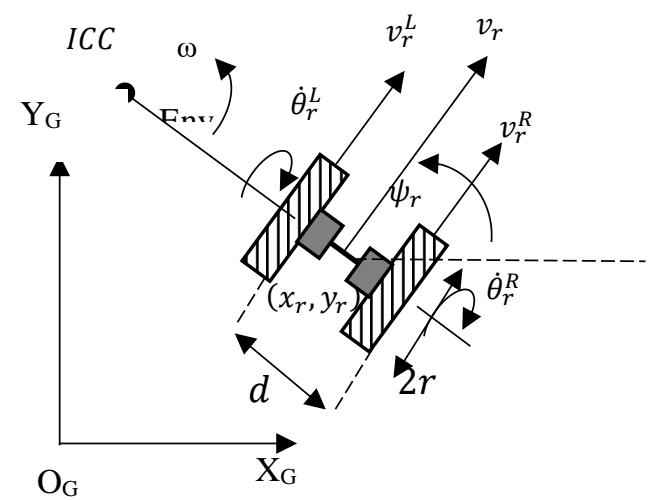

Figure.9 Configuration of mobile robot

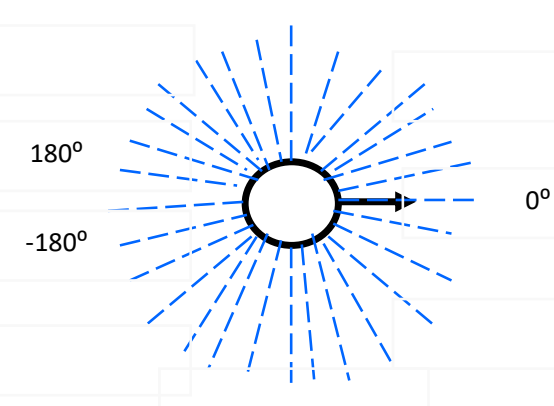

Figure.10 Configuration of LIDAR's scan angles 
This study proposes modified terms in sum of repulsive forces that are different from pedestrian's interaction $\mathbf{f}_{i}^{e}=\mathbf{f}_{i}^{p}+\mathbf{f}_{i}^{w}$ in [13] that include repulsive forces $\mathbf{f}_{i}^{p}$ exerted on individual $i$ by other pedestrian and by stationary obstacles in environment $\mathbf{f}_{i}^{w}$ such as walls. This study assumes that the sum of repulsive force $\sum \mathbf{F}_{r-o b s}^{r e p}$ are affected not only by static obstacle objects $\mathbf{F}_{r-s o}^{\text {rep }}$ and other single static or moving human $\mathbf{F}_{r-s h}^{r e p}$, but also influenced by humanobject interaction $\mathbf{F}_{r-h o i}^{r e p}$ and interaction with group of human $\mathbf{F}_{r-g h}^{r e p}$ as described as follows:

$$
\begin{aligned}
\sum \mathbf{F}_{r-o b s}^{r e p}= & \mathbf{F}_{r-s o}^{r e p}+\mathbf{F}_{r-s h}^{r e p}+\mathbf{F}_{r-h o i}^{r e p} \\
& +\mathbf{F}_{r-g h}^{r e p}
\end{aligned}
$$

Repulsive force of static obstacle objects is described as follows:

$$
\begin{aligned}
& \mathbf{F}_{h-s o}^{r e p} \\
& =K_{h}^{s t r} \exp \left(\frac{\left(r_{h-s o}-d_{h-s o}\right)}{K_{h}^{r n g}}\right) \boldsymbol{n}_{h-s o} \\
& +K_{h}^{c m p} \mathrm{~g}\left(r_{h-s o}-d_{h-s o}\right) \boldsymbol{n}_{h-s o} \\
& +K_{h}^{f r i} \mathrm{~g}\left(r_{h-s o}-d_{h-s o}\right) \Delta v_{s o-h}^{t} \mathbf{t}_{h-s o}
\end{aligned}
$$

Repulsive force of single static or moving human is formulated as follows:

$$
\begin{aligned}
& \mathbf{F}_{h-s h}^{r e p} \\
& =K_{h}^{s t r} \exp \left(\frac{\left(r_{h-s h}-d_{h-s h}\right)}{K_{h}^{r n g}}\right) \boldsymbol{n}_{h-s h} \\
& +K_{h}^{c m p} \mathrm{~g}\left(r_{h-s h}-d_{h-s h}\right) \boldsymbol{n}_{h-s h} \\
& +K_{h}^{f r i} \mathrm{~g}\left(r_{h-s h}-d_{h-s h}\right) \Delta v_{s h-h}^{t} \mathbf{t}_{h-s h}
\end{aligned}
$$

Repulsive force of human-object interaction is expressed as follows:

$$
\begin{aligned}
& \mathbf{F}_{h-h o i}^{r e p} \\
& =K_{h}^{s t r} \exp \left(\frac{\left(r_{h-h o i}-d_{h-h o i}\right)}{K_{h}^{r n g}}\right) \boldsymbol{n}_{h-h o i} \\
& +K_{h}^{c m p} \mathrm{~g}\left(r_{h-h o i}-d_{h-h o i}\right) \boldsymbol{n}_{h-h o i} \\
& +K_{h}^{\text {fri }} \mathrm{g}\left(r_{h-h o i}\right. \\
& \left.-d_{h-h o i}\right) \Delta v_{h o i-h}^{t} \mathbf{t}_{h-h o i}
\end{aligned}
$$

Repulsive force as result of interaction with human group is described as follows:

$$
\begin{aligned}
& \mathbf{F}_{h-g h}^{r e p} \\
& =K_{h}^{s t r} \exp \left(\frac{\left(r_{h-g h}-d_{h-g h}\right)}{K_{h}^{r n g}}\right) \boldsymbol{n}_{h-g h} \\
& +K_{h}^{c m p} \mathrm{~g}\left(r_{h-g h}-d_{h-g h}\right) \boldsymbol{n}_{h-g h} \\
& +K_{h}^{f r i} \mathrm{~g}\left(r_{h-g h}-d_{h-g h}\right) \Delta v_{g h-h}^{t} \mathbf{t}_{h-g h}
\end{aligned}
$$

Attractive force $\mathbf{F}_{r-t a r}^{\text {att }}$ that motivate robot to approach target is defined as follows:

$$
\mathbf{F}_{r-t a r}^{a t t}=K_{r}^{v}\left(v_{r}^{\text {des }}-v_{r}^{a c t}\right)
$$

Robot will move to target direction by using desired velocity $v_{r}^{d e s}$. Time-constant $K_{r}^{v}$ is needed for mobile robot to move from current actual velocity $v_{r}^{a c t}$ to desired velocity $v_{r}^{\text {des }}$. The desired velocity used to be defined by giving certain value or by adjusting to random number [13]. This research proposes to utilize HVO [12] to generate desired velocity based on LIDAR scan on robot surrounding as described as follows:

$$
\begin{gathered}
H V O=\bigcup_{o \in O} V O_{r \mid o} \cup \bigcup_{h \in P} R V O_{r \mid r o} \\
\cup \bigcup_{\text {ro }} N L V O_{r \mid h} \\
V_{\text {safe }_{\Omega}}=\overline{H V O_{\Omega}}=\left\{v_{r} \in \mathcal{V} \mid v_{r} \notin H V O_{\Omega}\right\} \\
v_{r}^{\text {des }}=\arg \min _{v_{r}^{\text {hvo* }} \in V_{\text {safe }}}\left(v_{\text {max }}^{c a}, v_{r}^{\text {hvo* }}\right)
\end{gathered}
$$

By calculating resultant of HVO-modHSFM forces, change on robot velocity can be determined as follows:

$$
\begin{gathered}
a_{r}(k)=\frac{1}{m_{r}} \mathbf{F}_{r}^{H V O-\operatorname{modHSFM}}(k) \\
v_{c m d}^{c a}=v_{r}^{*}(k)=v_{r}(k)+a_{r}(k) T
\end{gathered}
$$

where acceleration of mobile robot $a_{r}(k)$ can be obtained by providing robot body mass $m_{r}$ and total force $\mathbf{F}_{r}^{H V O-m o d H S F M}(k)$ that act on robot. The actual velocity of robot is denoted by $v_{r}(k)$. Discrete time interval while acceleration $a_{r}(k)$ happen is symbolized by $T$. The new actual linear velocity of robot is expressed by $v_{r}^{*}(k)$.

The angular velocity is determined by utilizing LIDAR scan as described as follows: 


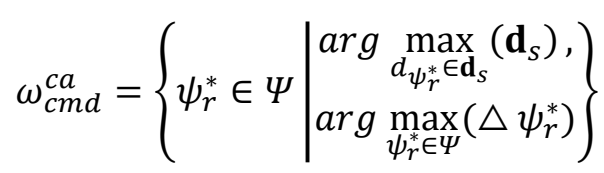

\section{Simulation results}

For demonstrating the effectiveness of proposed method to avoid collision with disturbed groups of pedestrians while navigates in indoor human environment, HVO-based modified HSFM is compared to HSFM, VFH, VO, RVO, APF, MSVO, and $\mathrm{HVO}$ from previous studies by using following scenarios.

The first scenario tested collision avoidance method to avoid a group of pedestrians GP, single pedestrian SP, F-Formation FF and individual human interacts with static object HOI. In this scenario, a group of pedestrians GP consisted of five peoples and F-Formation FF involved three peoples. All human objects in this simulation were symbolized by circles. Each object had its own label. Position of mobile robot and pedestrians were represented by the center of circles. Orientation of each object was visualized by using red short straight line that drawn from circle's center. Static object was represented by a square.

The second scenario evaluated the ability of collision avoidance method to avoid two disturbed groups of pedestrians, single pedestrian, and two static humans interact with static object. The first group of disturbed pedestrians DGP consisted of five members and the second group of disturbed pedestrians DG2P consisted of two members. Both groups of pedestrians in second scenario walked with velocity that faster than velocity of a group of pedestrians in the first scenario.

The diameter of mobile robot MR $d=0.6 \mathrm{~m}$ and wheel radii $r=0.15 \mathrm{~m}$ with desired linear velocity $\mathbf{v}_{c m d}^{p f}=1.0 \mathrm{~m} / \mathrm{s}$. Each human as static or moving pedestrian had diameter of body $d_{h}=0.6 \mathrm{~m}$. Target position of walking human are represented by points. Static pedestrian ST interacted with static object target at STT. All pedestrians were equipped with HSFM algorithm for representing walking behavior of human. In the first scenario, single pedestrian SP walked with velocity of $0.5 \mathrm{~m} / \mathrm{s}$ to SPT position and each member of group pedestrians GP moved with desired linear velocity of $1.0 \mathrm{~m} / \mathrm{s}$ to GPT location. In the second scenario, each member of the first disturbed group of pedestrians DGP moved with velocity of $1.5 \mathrm{~m} / \mathrm{s}$ to GPT location and each member of the second disturbed group of pedestrians DG2P moved with velocity of $1.4 \mathrm{~m} / \mathrm{s}$ to G2PT location. In both scenarios, F-Formation FF and human object interaction $\mathrm{HOI}$ represented static pedestrians.

PRM were utilized to generate random paths of mobile robot, single pedestrian, and group of pedestrians in each simulation as shown in Fig. 11. These paths were created based on map with dimension $15 \times 15 \mathrm{~m}^{2}$ of robot workspace that represented hall of indoor environment. The border of this environment was visualized with black bold lines. Initial and target position of each object in simulation was configured in such a way to create crossing condition.

\subsection{First Scenario}

Indoor navigation system of mobile robot with respect to its kinematics was used for simulation with HSFM, VFH, VO, RVO, APF, MSVO, and HVO. Whereas the proposed method, HVO-based modified HSFM, was utilized in navigation system with respect to kinematics and dynamics of mobile robot. The complete mobile robot trajectory of each simulation is depicted in Fig. 12 as red winding line.
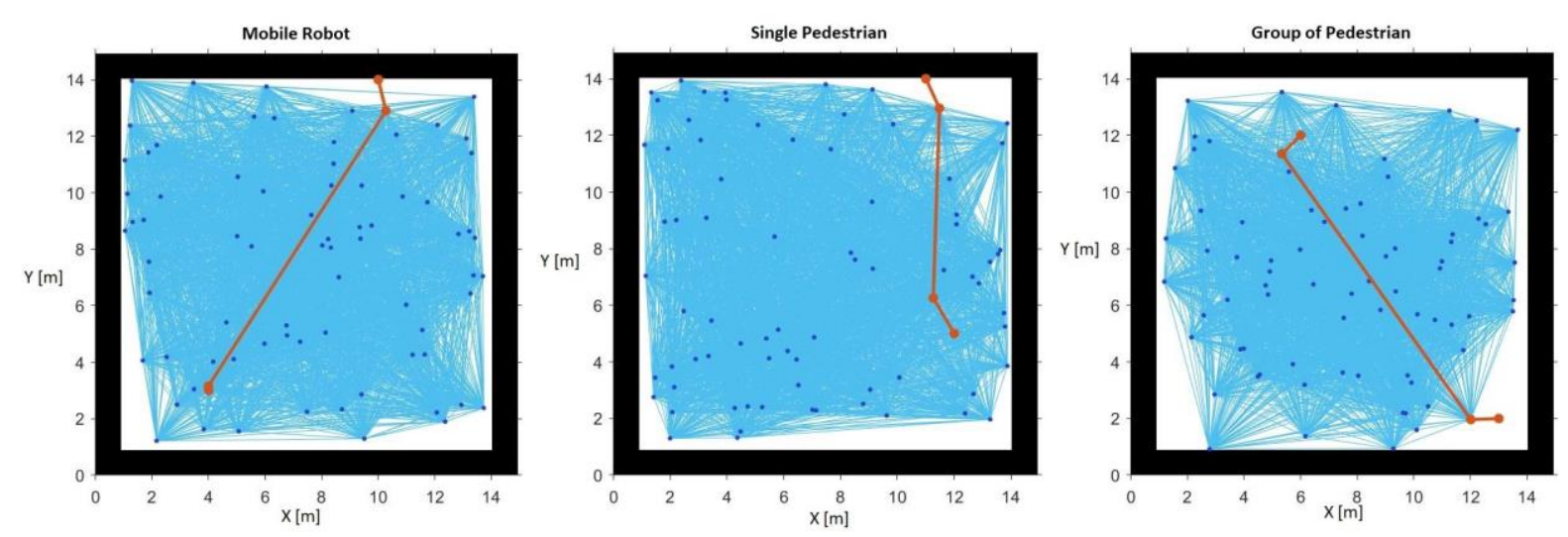

Figure.11 PRM-based random paths of mobile robot, single pedestrian, and group of pedestrians 
Trajectories of pedestrians are drawn as blue dash winding lines. LIDAR scans are symbolized by blue dash straight lines that come out of mobile robot center. The last configuration of all objects in simulation can be viewed in this figure. Comparison of reference and actual trajectories are shown in Fig. 13. Reference trajectory is illustrated as blue line. Actual trajectory is drawn as red line. Safety and comfort performance of simulations in the scenario 1 are shown in Fig. 14.

In the first simulation, HSFM scored 0.44 of TLI with group pedestrians GP. In the second simulation, Robot with VFH moved to close to pedestrians by scoring $0.33,0.64$, and 0.67 of $T L I$ with GP, FF, and HOI respectively. VO and RVO used in the third and fourth simulations by achieving 0.5 of $T L I$ with GP. In the fifth simulation, APF obtained 0.57 of $T L I$ while robot manoeuvred in close distance with HOI. In the sixth simulation, comfort of GP was distracted by robot moved with MSVO by scoring 0.34 of TLI. In the seventh simulation, HVO can maintain safety and comfort of GP with 0.24 of TLI. Max of TLI, 0.51, was resulted because of collision avoidance with HOI. In the eighth simulation, HVO-based modified HSFM can maintain safety and comfort of humans including group of pedestrians GP with 0.16 of $T L I$.

\subsection{Second Scenario}

HSFM, VFH, VO, RVO, APF, MSVO, and HVO were applied to indoor navigation system of mobile robot respectively by concerning to its kinematics to avoid two disturbed groups of pedestrians, single pedestrian, a group of F-formation consisted of three persons, and human object interaction. The proposed method, HVO-based modified HSFM, was implemented in indoor navigation system with respect to mobile robot's kinematics and dynamics.

Mobile robot trajectory from initial to target position from each simulation in scenario 2 is shown in Fig. 15. Reference trajectories to be tracked and actual trajectories as results of tracking and collision avoidance are compared in Fig. 16. The proposed method, HVO-based modified HSFM, and other methods are compared in the ability to maintain safety and comfort of pedestrians by using TLI. The results of these comparisons are visualized in Fig. 17.

In the ninth simulation, robot with HSFM threatened disturbed group pedestrians that consisted of two persons, DG2P and resulted 0.82 of TLI. In the tenth simulation, VFH scored 0.45 of $T L I$ with DG2P. VO achieved 0.5 of $T L I$ with DG2P in the eleventh simulation. In the twelfth simulation, RVO moved to close to DG2P with 0.5 of TLI. APF endangered SP and obtained 0.9 of $T L I$ in the thirteenth simulation.
Safety of DG2P was threatened by robot moved with MSVO in the fourteenth simulation by scoring 0.69 of TLI. The maximum TLI of HVO-based collision avoidance is 0.75 with respect to single pedestrian SP, in the fifteenth simulation. This simulation resulted in collision avoidance that can maintain safety and comfort of first disturbed group of pedestrians DGP with 0.12 of TLI. This value describes that robot moved to close to single pedestrian SP while it avoided collision with a member of the first group of disturbed pedestrians DGP. Mobile robot that had to maneuver for collision avoidance made actual trajectory had more length of $14.8 \mathrm{~m}$ than reference trajectory with length of $11.93 \mathrm{~m}$. In the sixteenth simulation, HVO-based modified HSFM scored TLI value of 0.18 when robot avoided two disturbed groups of pedestrians. Actual trajectory with collision avoidance movement had more length of $19.24 \mathrm{~m}$ than reference trajectory with length of $12.50 \mathrm{~m}$.

Table 2 summarizes results of simulations to compare the distance of travelled trajectories that affected by HSFM, VFH, VO, RVO, APF, MSVO, $\mathrm{HVO}$, and HVO-based modified HSFM in two scenarios. Table 3 reports comparison of TLI as performance evaluation of HSFM, VFH, VO, RVO, APF, MSVO, HVO, and HVO-based modified HSFM in two scenarios. The first scenario includes pedestrians with normal velocities. While the second scenario involves disturbed groups of pedestrians with faster velocities. From this summary of collision avoidance performance evaluation, HVO-based modified HSFM has the lowest score of TLI. It means that the proposed collision avoidance method is capable to maintain safety and comfort of groups of pedestrians while navigate to target location in both scenarios.

\section{Conclusion}

The aim of this study was to improve HVO in order to be able to handle complex situation in human environment with disturbed groups of pedestrians by presenting HVO-based modified HSFM with respect to kinematics and dynamics of mobile robot. The originality of this work is based on the modification of HSFM by presenting human-object interaction term and group of pedestrian term with adaptation of HVO to handle moving objects that move with unknown trajectory and with changeable velocities. Trajectory of each moving object in simulation is randomly generated by applying modified PRM to get unique and more realistic objects movements. 

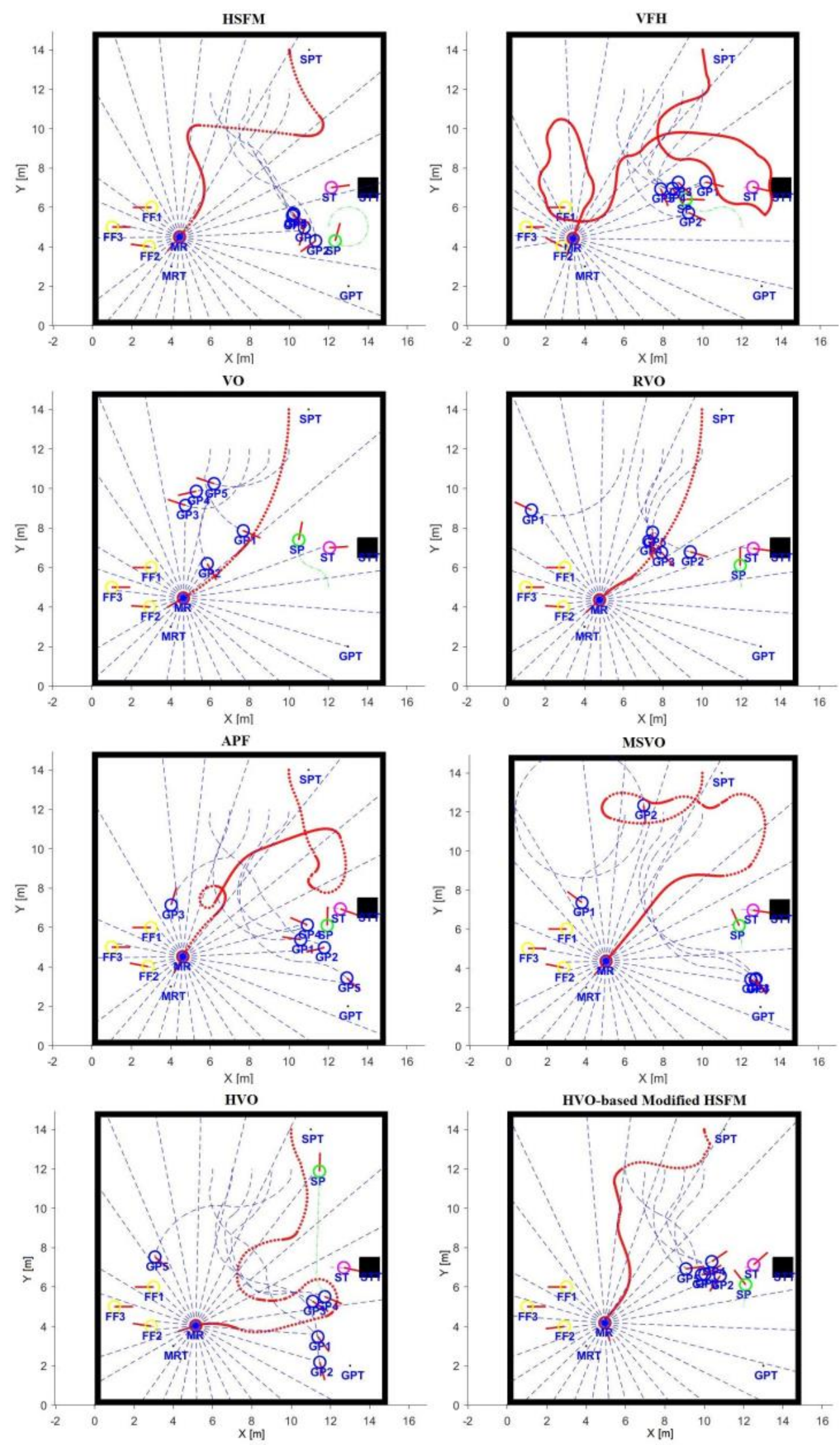

Figure.12 Trajectory of mobile robot that avoid collision in scenario 1 using HSFM, VFH, VO, RVO, APF, MSVO, HVO, and HVO-based Modified HSFM respectively 
HSFM

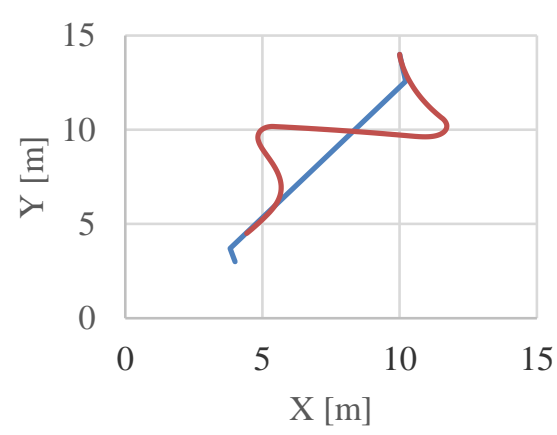

VO

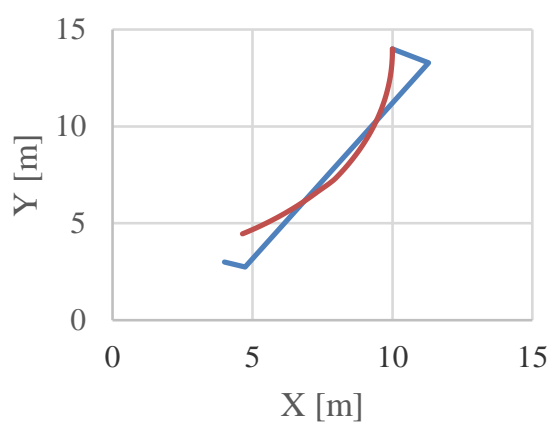

APF

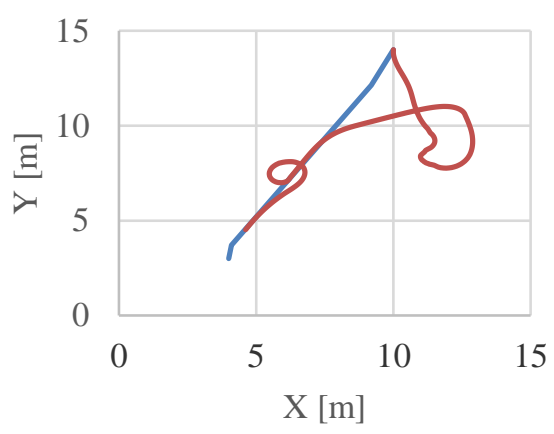

HVO

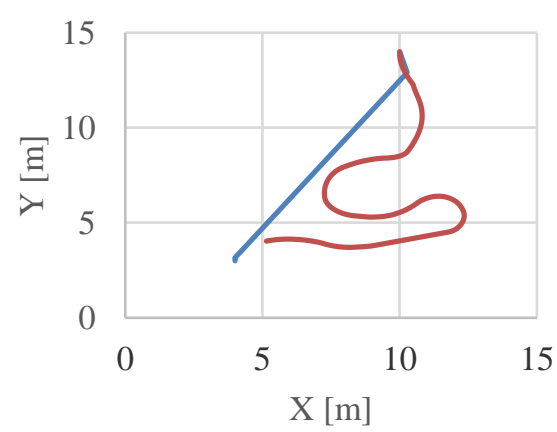

VFH



RVO

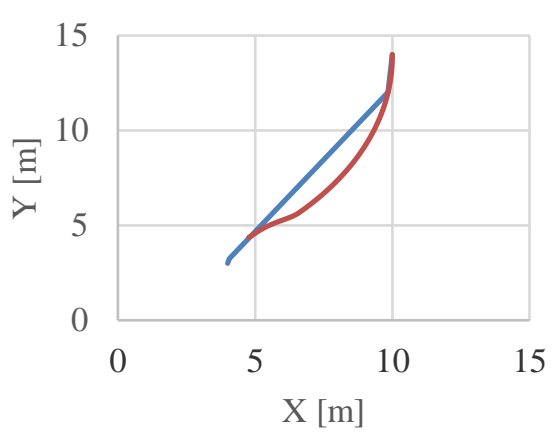

MSVO

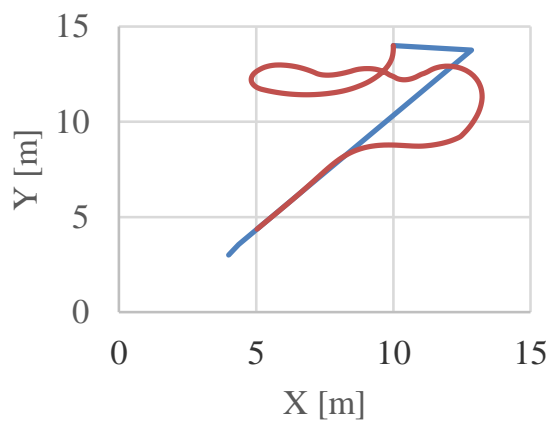

HVO-based Modified HSFM

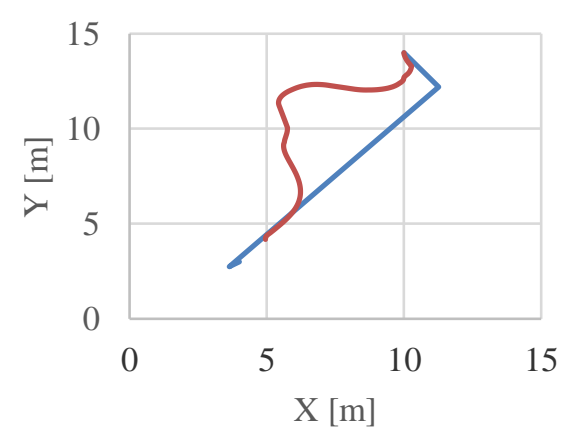

Figure. 13 Reference and actual trajectories comparison of mobile robot that avoid collision in scenario 1 using HSFM. VFH. VO. RVO. APF. MSVO. HVO. and HVO-based Modified HSFM respectivelv 
HSFM

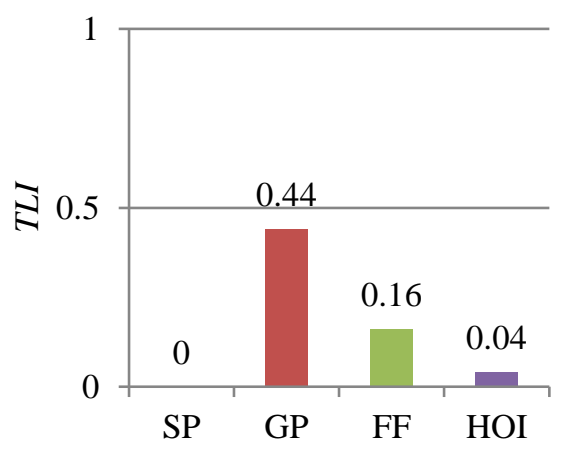

VO

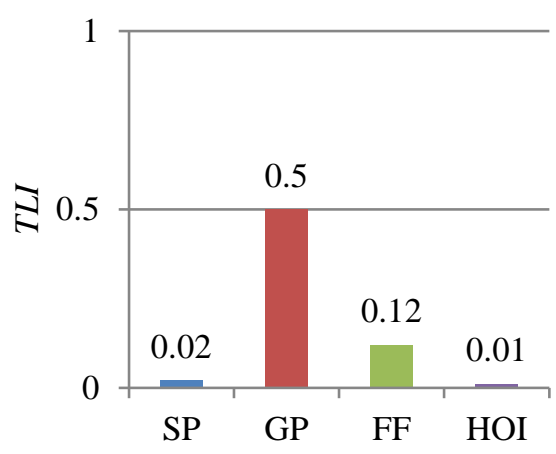

APF

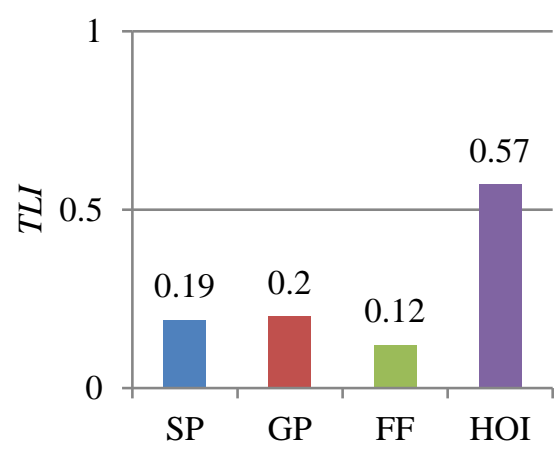

HVO

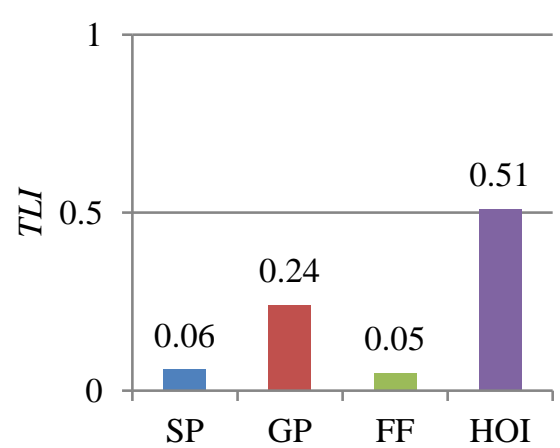

VFH

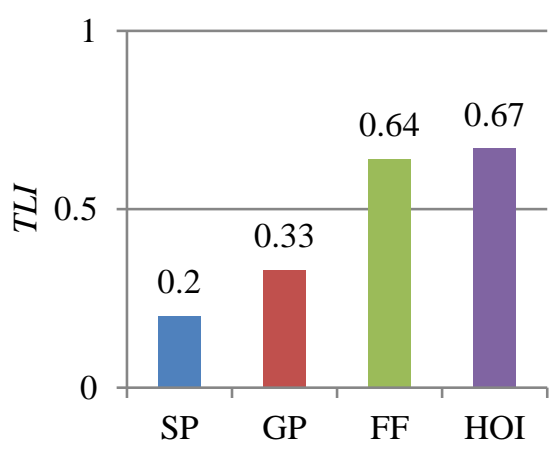

RVO

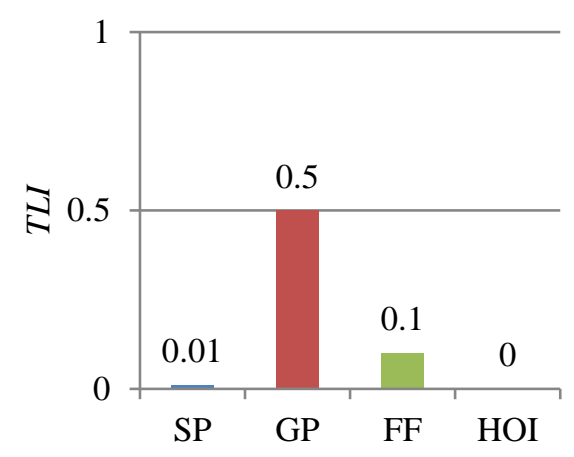

MSVO

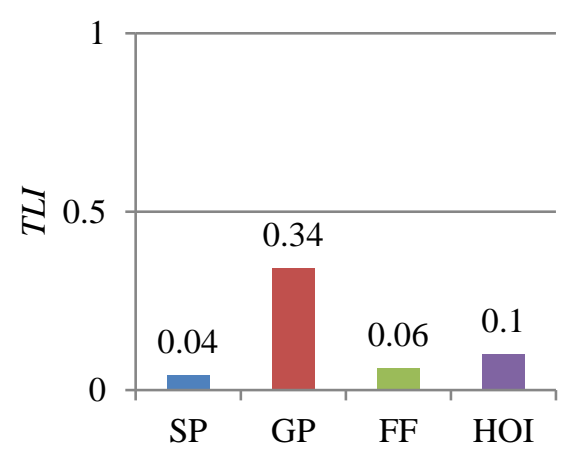

HVO-based modified HSFM

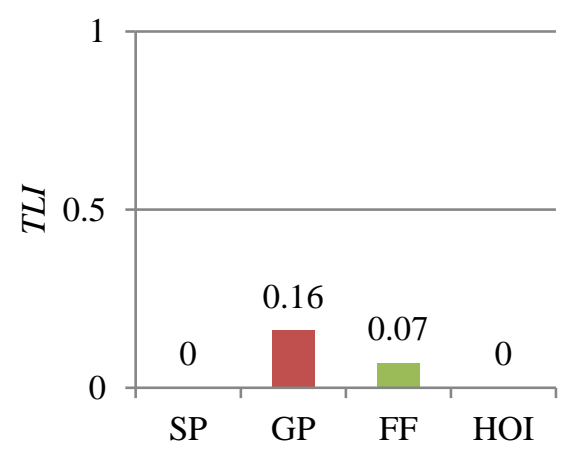

Figure. 14 Safety and comfort performance evaluation on HSFM, VFH, VO, RVO, APF, MSVO, HVO, and HVO-based modified HSFM respectively in scenario 1 



Figure.15 Trajectory of mobile robot that avoid collision in scenario 2 using HSFM, VFH, VO, RVO, APF, MSVO, HVO, and HVO-based Modified HSFM respectively

Switching strategy is implemented in mobile robot and other moving objects in these simulations by pairing pure pursuit algorithm and collision avoidance method. This work also proposes the use of TLI index to measure safety and comfort of human that exist in the vicinity of mobile robot.

The proposed HVO-based modified HSFM has been tested to avoid static and moving pedestrians. There are two scenarios that involve human-object interaction, individual pedestrian, and groups of pedestrians with different velocities have been conducted in simulations.
Results of simulations show that HVO-based modified HSFM has the ability to maintain safety and comfort of static and moving pedestrians while it operates in human environment. In this study, $T L I$ is used as indicator to measure performance of several collision avoidance methods while facing normal and faster pedestrians that move with changeable velocities.

Based on these simulations, the proposed collision avoidance method, HVO-based modified HSFM, gains the lowest TLI value compare to other methods by the score of 0.14 in average by concerning safety and comfort of groups of 
HSFM



Vo

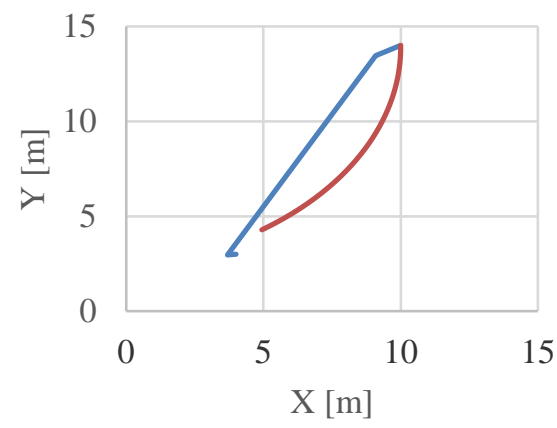

APF

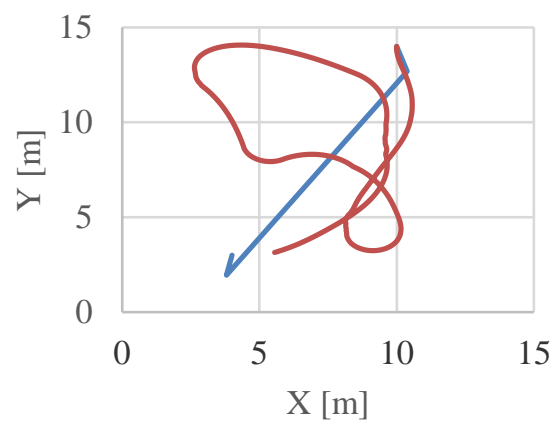

HVO



VFH

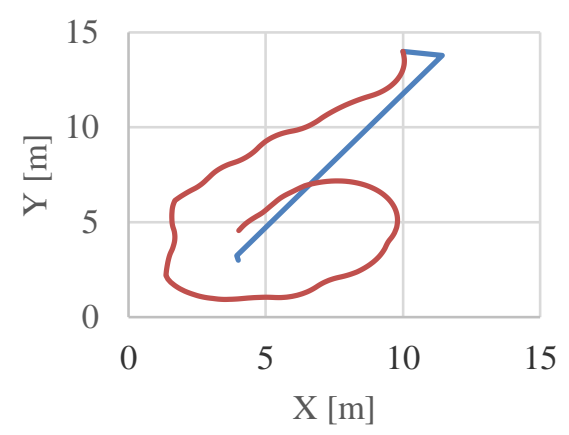

RVO

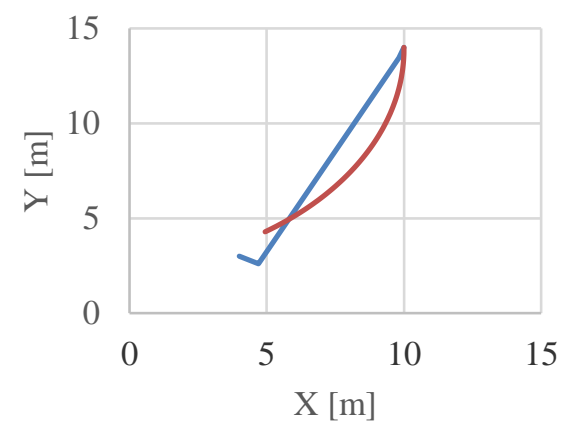

MSVO

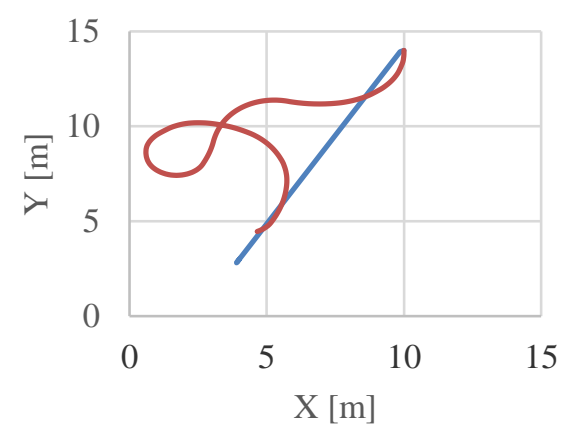

HVO-based Modified HSFM

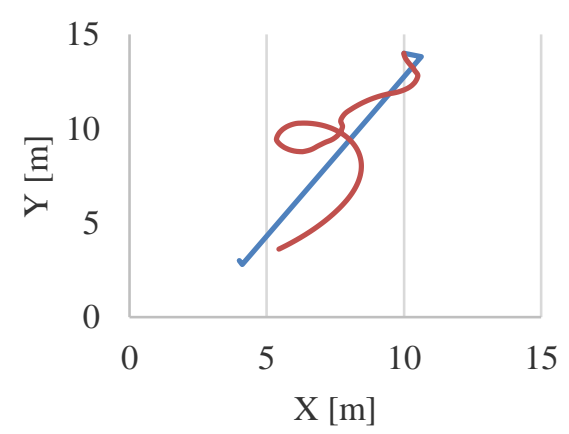

Figure.16 Reference and actual trajectories comparison of mobile robot that avoid collision in scenario 2 using HSFM, VFH, VO, RVO, APF, MSVO, HVO, and HVO-based Modified HSFM respectively 
HSFM

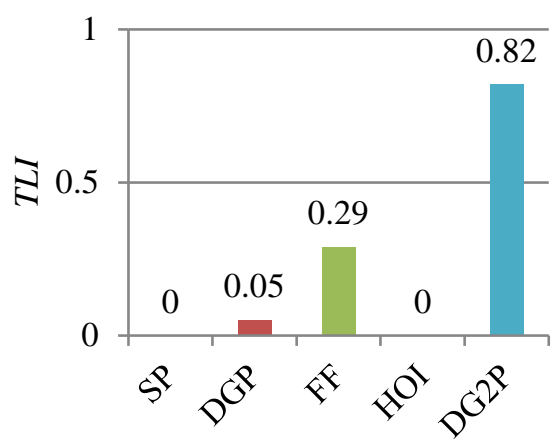

Vo



APF

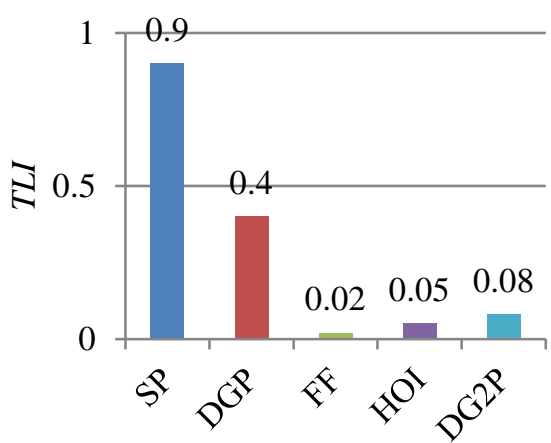

HVO

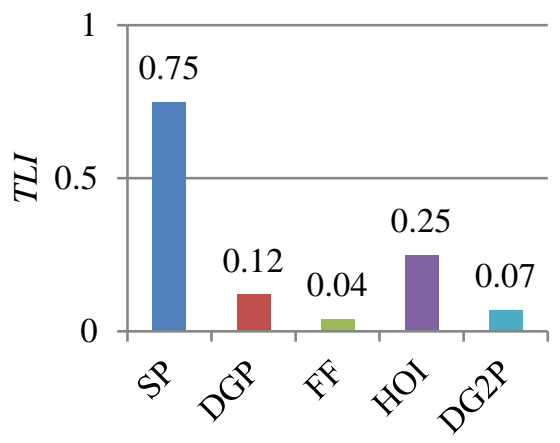

VFH

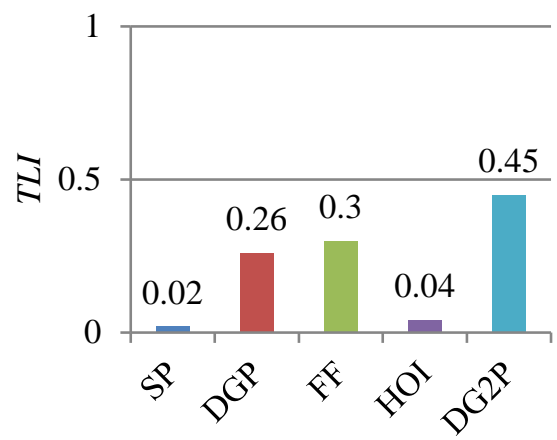

RVO

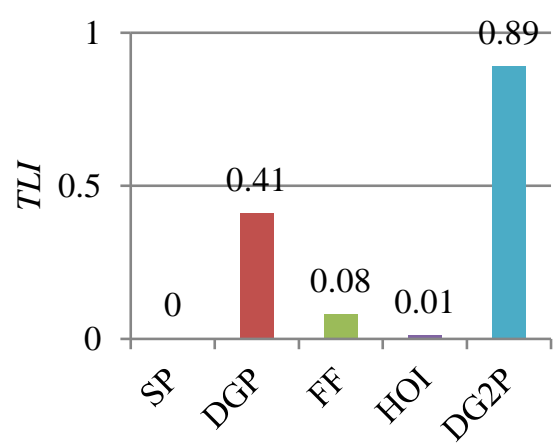

MSVO

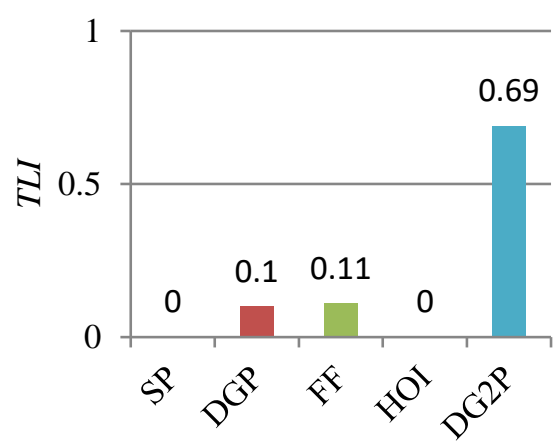

HVO-based Modified HSFM



Figure.17 Safety and comfort performance evaluation on HSFM, VFH, VO, RVO, APF, MSVO, HVO, and HVO-based Modified HSFM respectively in scenario 2 
Table 2. Comparison of reference and travelled trajectories affected by collision avoidance simulations based on HSFM, VFH, VO, RVO, APF, MSVO, HVO, and HVO-based modified HSFM

\begin{tabular}{|l|c|c|c|c|c|c|c|c|}
\hline & HSFM & VFH & VO & RVO & APF & MSVO & HVO & $\begin{array}{c}\text { HVO- } \\
\text { based } \\
\text { modified } \\
\text { HSFM }\end{array}$ \\
\hline $\begin{array}{l}\text { Reference } \\
\text { trajectory } \\
\text { distances } \\
\text { in } \\
\text { Scenario 1 } \\
\text { [m] }\end{array}$ & 11.91 & 14.60 & 13.47 & 11.55 & 11.39 & 15.60 & 11.68 & 13.59 \\
\hline $\begin{array}{l}\text { Travelled } \\
\text { distances } \\
\text { in } \\
\begin{array}{l}\text { Scenario 1 } \\
\text { [m] }\end{array}\end{array}$ & & & & & & & \\
\hline $\begin{array}{l}\text { Reference } \\
\text { trajectory } \\
\text { distances } \\
\text { in } \\
\text { Scenario 2 } \\
\text { [m] }\end{array}$ & 17.42 & 42.60 & 11.50 & 11.50 & 25.59 & 29.00 & 24.20 & 14.48 \\
\hline $\begin{array}{l}\text { Travelled } \\
\text { distances } \\
\text { in } \\
\text { Scenario 2 } \\
\text { [m] }\end{array}$ & 12.68 & 13.43 & 11.98 & 12.18 & 13.83 & 11.78 & 11.93 & 12.50 \\
\hline
\end{tabular}

Table 3. Comparison of TLI of collision avoidance simulations based on HSFM, VFH, VO, RVO, APF, MSVO,

\begin{tabular}{|c|c|c|c|c|c|c|c|c|}
\hline \multirow[t]{2}{*}{ Simulation } & \multicolumn{8}{|c|}{ Threat Level Index $(T L I)$} \\
\hline & HSFM & VFH & VO & RVO & $\mathbf{A P F}$ & MSVO & HVO & $\begin{array}{c}\text { HVO- } \\
\text { based } \\
\text { modified } \\
\text { HSFM }\end{array}$ \\
\hline $\begin{array}{l}\text { Scenario } 1 \\
\text { with } \\
\text { normal } \\
\text { velocity of } \\
\text { pedestrians }\end{array}$ & 0.44 & 0.33 & 0.50 & 0.50 & 0.20 & 0.34 & 0.24 & 0.16 \\
\hline $\begin{array}{l}\text { Scenario } 2 \\
\text { with } \\
\text { disturbed } \\
\text { groups of } \\
\text { pedestrians }\end{array}$ & 0.44 & 0.36 & 0.44 & 0.65 & 0.24 & 0.40 & 0.10 & 0.13 \\
\hline $\begin{array}{l}\text { Average of } \\
T L I\end{array}$ & 0.44 & 0.34 & 0.47 & 0.58 & 0.22 & 0.37 & 0.17 & 0.14 \\
\hline
\end{tabular}

pedestrians. For future research, we will utilize HVObased modified HSFM into real transport mobile robot navigation system in order to navigate safely and comfort in human environment.

\section{Conflicts of Interest}

The authors declare no conflict of interest. 


\section{Author Contributions}

Conceptualization, M.F.; methodology, M.F.; software, M.F.; validation, M.F.; formal analysis, M.F.; investigation, M.F.; resources, M.F.; data curation, M.F.; writing - original draft preparation, M.F.; writing - review and editing, M.F., T.A., and D.P.; visualization, M.F.; supervision, T.A. and D.P.

\section{References}

[1] ASME RPPTF, "Robotics \& COVID-19: How Robotic Technologies are Critical in Getting America Up and Running in The Age of the COVID-19 Pandemic", The American Society of Mechanical Engineers Robotics Public Policy Task Force white paper, 2020.

[2] F. Rubio, F. Valero, C. Llopis-Albert, "A Review of Mobile Robots: Concepts, Methods, Theoretical Framework, and Applications", International Journal of Advanced Robotic Systems, Vol. 16, No. 2, pp. 1-22, 2019.

[3] F. Fang, M. Shi, K. Qian, B. Zhou, Y. Gan, “A Human-aware Navigation Method for Social Robot Based on Multi-layer Cost Map", International Journal of Intelligent Robotics and Applications 4, pp. 308-318, 2020.

[4] X. Fan, Y. Guo, H. Liu, B. Wei, W. Lyu, "Improved Artificial Potential Field Method Applied for AUV Path Planning", Mathematical Problems in Engineering, Vol. 2020, Article ID 6523158, pp. 1-21, 2020.

[5] A. Azzabi, K. Nouri, "An Advanced Potential Field Method Proposed for Mobile Robot Path Planning", Transactions of the Institute of Measurement and Control, Vol. 41, No. 11, pp. 3132-3144, 2019.

[6] A. Babinec, F. Duchoň, M. Dekan, Z. Mikulová, L. Jurišica, "Vector Field Histogram* with Look-ahead Tree Extension Dependent on Time Variable Environment", Transactions of the Institute of Measurement and Control, Vol. 40, No. 4, pp. 1250-1264, 2018.

[7] H. Kim, U.K. Cheang, M. J. Kim, “Autonomous Dynamic Obstacle Avoidance for Bacteriapowered Microrobots (BPMs) with Modified Vector Field Histogram", PLoS ONE, Vol. 12, No. 10, pp. 1-23, 2017.

[8] M. Fuad, T. Agustinah, and D. Purwanto, "Autonomous Indoor Vehicle Navigation Using Modified Steering Velocity Obstacles", In: Proc. of 2020 International Seminar on Intelligent Technology and Its Applications (ISITIA), Surabaya, Indonesia, pp. 83-88, 2020.

[9] W. Wu, H. Jia, Q. Luo, Z. Wang, "Dynamic Path Planning for Autonomous Driving on
Branch Streets with Crossing Pedestrian Avoidance Guidance", IEEE Access, Vol. 7, pp. 144720-144731, 2019.

[10] L. Sun, J. Zhai and W. Qin, "Crowd Navigation in an Unknown and Dynamic Environment Based on Deep Reinforcement Learning", IEEE Access, Vol. 7, pp. 109544-109554, 2019.

[11] S. Zheng and H. Liu, "Improved Multi-Agent Deep Deterministic Policy Gradient for Path Planning-Based Crowd Simulation", IEEE Access, Vol. 7, pp. 147755-147770, 2019.

[12] M. Fuad, T. Agustinah, D. Purwanto, "Collision Avoidance of Multi Modal Moving Objects for Mobile Robot Using Hybrid Velocity Obstacles", International Journal of Intelligent Engineering and Systems, Vol. 13, No. 3, pp. 407-421, 2020.

[13] F. Farina, D. Fontanelli, A. Garulli, A. Giannitrapani, and D. Prattichizzo, "Walking Ahead: The Headed Social Force Model", PLoS ONE, Vol. 12, No. 1, pp. 1-23, 2017.

[14] D. Brockmann, L. Hufnagel, and T. Geisel, "The Scaling Laws of Human Travel", Nature 439, pp. 462-465, 2006.

[15] M. Moussaid, N. Perozo, S. Garnier, D. Helbing, and G. Theraulaz, "The walking behaviour of pedestrian social groups and its impact on crowd dynamics", PLoS ONE, Vol. 5, No. 4, pp. 17,2010 .

[16] D. Helbing, P. Molnár, I.J. Farkas, and K. Bolay, "Self-Organizing Pedestrian Movement", Environment and Planning B: Planning and Design, Vol. 28, No. 3, pp. 361-383, 2001.

[17] G.Arechavaleta, J. Laumond, H. Hicheur, and A. Berthoz, "On the Nonholonomic Nature of Human Locomotion", Autonomous Robots 25, pp. 25-35, 2008.

[18] E. T. Hall, The Hidden Dimension: Man's Use of Space in Public and Private. London, U.K.: The Bodley Head Ltd, 1966.

[19] T. M. Ciolek and A. Kendon, "Environment and the Spatial Arrangement of Conversational Encounters", Sociological Inquiry, Vol. 50, No. 3 - 4, pp. 237-271, 1980.

[20] P. McIlvenny, M. Broth, and P. Haddington, "Moving Together: Mobile Formations in Interaction", Space and Culture, Vol. 17, No. 2, pp. $104-106,2014$. 